\title{
Erratum to: Mass spectrometric screening and identification of acidic metabolites in fulvic acid fractions of contaminated groundwater
}

\author{
Carsten Jobelius • Fritz H. Frimmel • Christian Zwiener
}

Received: 21 May 2014 / Accepted: 21 May 2014 / Published online: 4 July 2014

(C) Springer-Verlag Berlin Heidelberg 2014

\section{Erratum to: Anal Bioanal Chem}

DOI 10.1007/s00216-014-7783-y

The authors would like to call the reader's attention to the following:

The second paragraph in the section "Elucidation of unknown structures" should read:

"A query for the chemical formula of peak $3\left(\mathrm{C}_{8} \mathrm{H}_{8} \mathrm{O}_{3}\right)$ in PubChem resulted in 633 hits. The fragmentation pattern of peak 3 reveals the loss of $\mathrm{CO}_{2}$ typical for aromatic or heterocyclic acids (Fig. 5a)".

In Table 3 two words were misspelled. They should correctly read: "Dihydrocarboxybenzothiophene" and "Benzofuranmethylsuccinic acid".

The online version of the original article can be found at http://dx.doi.org/ 10.1007/s00216-014-7783-y.

C. Jobelius $\cdot$ F. H. Frimmel

Water Chemistry, Engler-Bunte-Institut, Karlsruhe Institute of

Technology (KIT), 76131 Karlsruhe, Germany

C. Zwiener $(\square)$

Environmental Analytical Chemistry, Center for Applied

Geosciences (ZAG), Eberhard Karls Universität Tübingen,

72074 Tübingen, Germany

e-mail: christian.zwiener@uni-tuebingen.de 
Table 4 was unfortunately reproduced incorrectly. Below please find the corrected version:

Table 4 Identified (bold) and tentatively identified metabolites in the FA-fraction of the contaminated region (B22 FA). Additionally structural proposals (grey) for metabolites are listed

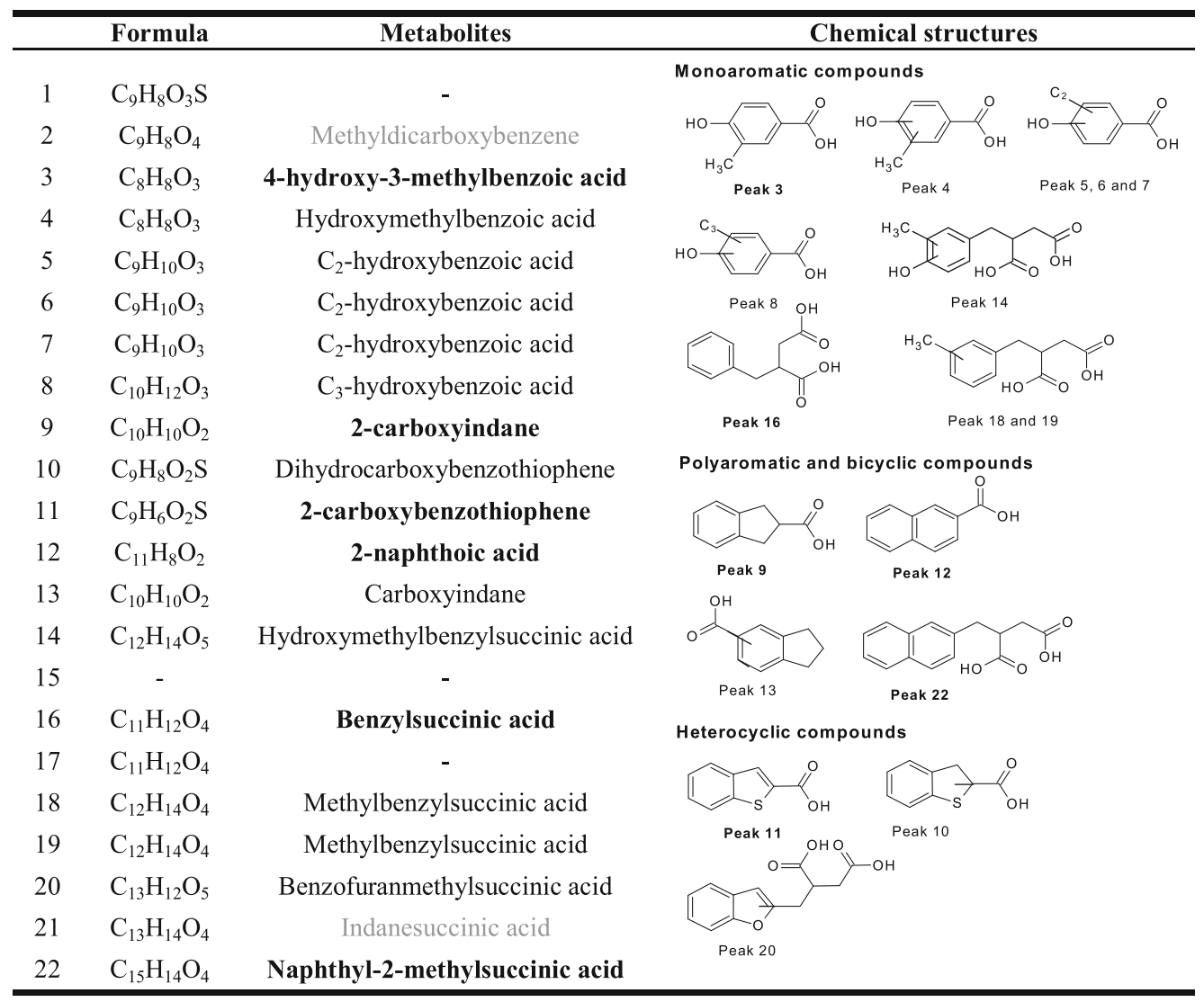

In the "Electronic Supplementary Material" the legend to Figure S2 should correctly read:

"Figure S2. (Part 3): LC-ESI-QTOF-MS fragmentation spectra (exemplarily at a collision energy of $\mathrm{CE}=10 \mathrm{eV}$ ) of the most intensive peaks derived from the NLS $\Delta \mathrm{m} / \mathrm{z}=44$. For peak 15 no fragmentation spectra could be obtained". 
In the "Electronic Supplementary Material" Table S1 was unfortunately reproduced incorrectly. Below please find the corrected version:

Table S1: Results of a literature search for acidic metabolites of monoaromatic, polycyclic aromatic, bicyclic and heterocyclic compounds found in batch experiments (b) and/or in the field (f)

\begin{tabular}{|c|c|c|c|c|}
\hline Metabolites & $\begin{array}{l}\text { Elemental } \\
\text { formulae }\end{array}$ & $\begin{array}{l}\text { (Possible) parent } \\
\text { compound }\end{array}$ & $\begin{array}{l}\text { Batch (b)/ } \\
\text { Field(f) }\end{array}$ & $\begin{array}{l}\text { Literature } \\
\text { (examples) }\end{array}$ \\
\hline \multicolumn{5}{|c|}{ of monoaromatic compounds (e.g. BTEX) } \\
\hline Benzoic acid & $\mathrm{C}_{7} \mathrm{H}_{6} \mathrm{O}_{2}$ & BTEX & (b), (f) & {$[1-5]$} \\
\hline Benzylsuccinic acid & $\mathrm{C}_{11} \mathrm{H}_{12} \mathrm{O}_{4}$ & Toluene & (b), (f) & {$[3,5-10]$} \\
\hline Benzylfumaric acid & $\mathrm{C}_{11} \mathrm{H}_{10} \mathrm{O}_{4}$ & Toluene & (b), (f) & [7] \\
\hline Methylbenzylsuccinic acid & $\mathrm{C}_{12} \mathrm{H}_{14} \mathrm{O}_{4}$ & Xylene & (b), (f) & {$[3,5-7,11,12]$} \\
\hline Methylbenzylfumaric acid & $\mathrm{C}_{12} \mathrm{H}_{12} \mathrm{O}_{4}$ & Xylene & (f) & [7] \\
\hline Methylbenzoic acid & $\mathrm{C}_{8} \mathrm{H}_{8} \mathrm{O}_{2}$ & Xylene & (f) & {$[3-5,13]$} \\
\hline Dimethylbenzoic acid & $\mathrm{C}_{9} \mathrm{H}_{10} \mathrm{O}_{2}$ & Trimethylbenzene & (b), (f) & {$[2,14]$} \\
\hline Phthalic acid & $\mathrm{C}_{8} \mathrm{H}_{6} \mathrm{O}_{4}$ & Xylene & (f) & [3] \\
\hline Trimethylbenzoic acid & $\mathrm{C}_{10} \mathrm{H}_{12} \mathrm{O}_{2}$ & Tetramethylbenzene & (f) & {$[13-15]$} \\
\hline $\mathrm{C}_{4}-$ to $\mathrm{C}_{6}$-benzoic acid & $\begin{array}{l}\mathrm{C}_{11} \mathrm{H}_{14} \mathrm{O}_{2} \\
\mathrm{C}_{13} \mathrm{H}_{18} \mathrm{O}_{2}\end{array}$ & $\mathrm{C}_{5^{-}}$to $\mathrm{C}_{7}$-benzene & (f) & [13] \\
\hline $\mathrm{C}_{2}$ - to $\mathrm{C}_{5}$-benzylsuccinic acid & $\begin{array}{l}\mathrm{C}_{13} \mathrm{H}_{16} \mathrm{O}_{4} \\
\mathrm{C}_{16} \mathrm{H}_{22} \mathrm{O}_{4}\end{array}$ & $\mathrm{C}_{3}$ - to $\mathrm{C}_{6}$-benzene & (f) & {$[13,16]$} \\
\hline Methylphenylacetic acid & $\mathrm{C}_{9} \mathrm{H}_{10} \mathrm{O}_{2}$ & & (f) & {$[14]$} \\
\hline \multicolumn{5}{|c|}{ of polycyclic aromatic hydrocarbons (PAH) and bicyclic compounds } \\
\hline Naphthyl-2-methylsuccinic acid & $\mathrm{C}_{15} \mathrm{H}_{14} \mathrm{O}_{4}$ & $\begin{array}{l}\text { Naphthalene, } \\
\text { 2-methylnaphthalene }\end{array}$ & (b), (f) & {$[4-6,17-19]$} \\
\hline Naphthoic acid & $\mathrm{C}_{11} \mathrm{H}_{8} \mathrm{O}_{2}$ & $\begin{array}{l}\text { Naphthalene, } \\
\text { Methylnaphthalene }\end{array}$ & (b), (f) & $\begin{array}{l}{[5,6,17,18} \\
20,21]\end{array}$ \\
\hline $\begin{array}{l}\text { Naphthyl-2-methylenesuccinic } \\
\text { acid }\end{array}$ & $\mathrm{C}_{15} \mathrm{H}_{12} \mathrm{O}_{4}$ & $\begin{array}{l}\text { Naphthalene, } \\
\text { 2-methylnaphthalene }\end{array}$ & (b), (f) & {$[17,19,22]$} \\
\hline $\begin{array}{l}\text { Tetra-, hexa-, octa- and } \\
\text { decahydronaphthoic acid }\end{array}$ & $\begin{array}{l}\mathrm{C}_{11} \mathrm{H}_{12} \mathrm{O}_{2} \\
\mathrm{C}_{11} \mathrm{H}_{18} \mathrm{O}_{2}\end{array}$ & $\begin{array}{l}\text { Naphthalene, } \\
\text { 2-Methylnaphthalene }\end{array}$ & (b), (f) & {$[4,5,17,23,24]$} \\
\hline Carboxycyclohexylacetic acid & $\mathrm{C}_{9} \mathrm{H}_{14} \mathrm{O}_{4}$ & $\begin{array}{l}\text { Naphthalene, } \\
\text { 2-methylnaphthalene }\end{array}$ & (b) & {$[25]$} \\
\hline Methylnaphthoic acid & $\mathrm{C}_{12} \mathrm{H}_{10} \mathrm{O}_{2}$ & 2-methylnaphthalene & (b), (f) & {$[4-6,24,26,27]$} \\
\hline $\begin{array}{l}\text { 1-methylnaphthyl-2-methyl- } \\
\text { succinic acid }\end{array}$ & $\mathrm{C}_{16} \mathrm{H}_{16} \mathrm{O}_{4}$ & 1-methylnaphthalene & (b) & {$[5,6,22]$} \\
\hline Naphthylacetic acid & $\mathrm{C}_{12} \mathrm{H}_{10} \mathrm{O}_{2}$ & Naphthalene & (f) & {$[18]$} \\
\hline Hydroxynaphthoic acid & $\mathrm{C}_{11} \mathrm{H}_{8} \mathrm{O}_{3}$ & Naphthalene & (f) & {$[18]$} \\
\hline Dimethylnaphthoic acid & $\mathrm{C}_{13} \mathrm{H}_{12} \mathrm{O}_{2}$ & & (b) & [28] \\
\hline Acenaphthenoic acid & $\mathrm{C}_{13} \mathrm{H}_{10} \mathrm{O}_{2}$ & Acenaphthene & (b) & {$[5,6,22]$} \\
\hline Acenaphthylmethylsuccinic acid & $\mathrm{C}_{17} \mathrm{H}_{16} \mathrm{O}_{4}$ & Acenaphthene & (b) & {$[5]$} \\
\hline Acenaphthylenoic acid & $\mathrm{C}_{13} \mathrm{H}_{8} \mathrm{O}_{2}$ & Acenaphthylene & (b) & {$[22]$} \\
\hline Phenanthrene carboxylic acid & $\mathrm{C}_{15} \mathrm{H}_{10} \mathrm{O}_{2}$ & Phenanthrene & (b) & {$[20]$} \\
\hline Fluorenoic acid & $\mathrm{C}_{14} \mathrm{H}_{10} \mathrm{O}_{2}$ & Fluorene & (f) & [5] \\
\hline Carboxyindane & $\mathrm{C}_{10} \mathrm{H}_{10} \mathrm{O}_{2}$ & Indane & (b), (f) & {$[5,22]$} \\
\hline Carboxyindene & $\mathrm{C}_{10} \mathrm{H}_{8} \mathrm{O}_{2}$ & Indene & (b), (f) & {$[5,22]$} \\
\hline \multicolumn{5}{|l|}{ of heterocyclic compounds } \\
\hline Carboxybenzothiophene & $\mathrm{C}_{9} \mathrm{H}_{6} \mathrm{O}_{2} \mathrm{~S}$ & Benzothiophene & (b), (f) & {$[4-6,22,29]$} \\
\hline Methylcarboxybenzothiophene & $\mathrm{C}_{10} \mathrm{H}_{8} \mathrm{O}_{2} \mathrm{~S}$ & Methylbenzothiophene & (f) & {$[6]$} \\
\hline Dihydrocarboxybenzothiophene & $\mathrm{C}_{9} \mathrm{H}_{8} \mathrm{O}_{2} \mathrm{~S}$ & Benzothiophene & (f) & {$[4,6,29]$} \\
\hline $\begin{array}{l}\text { Benzothiophenemethylsuccinic } \\
\text { acid }\end{array}$ & $\mathrm{C}_{13} \mathrm{H}_{12} \mathrm{O}_{4} \mathrm{~S}$ & Benzothiophene & (b), (f) & {$[6,22]$} \\
\hline $\begin{array}{l}\text { Benzothiophenemethylen- } \\
\text { succinic acid }\end{array}$ & $\mathrm{C}_{13} \mathrm{H}_{10} \mathrm{O}_{4} \mathrm{~S}$ & Benzothiophene & (b) & {$[22]$} \\
\hline Carboxybenzofuran & $\mathrm{C}_{9} \mathrm{H}_{6} \mathrm{O}_{3}$ & Benzofuran & (b), (f) & {$[5,6,22]$} \\
\hline Benzofuranmethylsuccinic acid & $\mathrm{C}_{13} \mathrm{H}_{12} \mathrm{O}_{5}$ & Benzofuran & (b) & {$[6,22]$} \\
\hline Benzofuranmethylensuccinic acid & $\mathrm{C}_{13} \mathrm{H}_{10} \mathrm{O}_{5}$ & Benzofuran & (b) & {$[22]$} \\
\hline Indolic acid & $\mathrm{C}_{9} \mathrm{H}_{7} \mathrm{O}_{2}$ & Indole & (b) & {$[22]$} \\
\hline
\end{tabular}

